\title{
Low-Intensity Alternating Electric Fields: A Potentially Safe and Effective Treatment of Cancer?
}

\author{
Thies Schroeder Benjamin L. Viglianti Mark W. Dewhirst \\ Department of Radiation Oncology, Duke University Medical Center, Durham, NC, USA
}

In this issue of Onkologie, Salzberg et al. [1] report the application of the novel cancer treatment modality using low-intensity, intermediate-frequency, alternating electric fields (Tumor-Treating Fields, TTF) for treatment of malignancies in human patients. This feasibility study has the explicit goal to demonstrate the safety and absence of adverse effects of this method in patients. The treatment is applied by electrodes patched onto the shaved skin of the patient, and powered by a mobile generator that is carried by the patient over a time span between 2 and over 4 weeks. The proposed main biological basis of this therapy is a perturbing effect of the alternating electrical fields on the formation of the mitotic spindle. A secondary effect could be a rupture of the membrane during mitosis, as observed in cultured cells and reported earlier [2]. As only mitotic cells would be affected, TTF is theoretically highly specific for cancer. The device for this therapy is marketed by the company NovoCure, which also funded both major publications that support this treatment $[2,3]$.

Using this treatment, Salzberg and coworkers report here a temporal regression of 3 out of 6 treated tumors, whereas the other patients showed partial responses or progressive disease. Apart from occasional skin irritations around the electrode contact area, no adverse reactions to the treatment were reported in this study. This is similar to the results of a separate pilot study that was conducted on patients with recurrent glioblastoma. This report also demonstrated good safety of the NovoTTF, with adverse effects consisting only of occasional skin irritation, similar to what was reported in Salzberg's paper. In the pilot study of recurrent glioblastoma, the overall survival of the group appeared to be superior to that of historical controls, but the study was small $(\mathrm{n}=10)$ and statistical comparisons were not feasible [3].

One of the major hurdles to overcome in performing treatments of this type is to establish a method to quantify the treatment that is being delivered. In this case, the authors have used finite element models to calculate the electric fields and in one case have verified the accuracy of this measurement in a volunteer [3]. Whereas this one case appears promising, it would seem that more detailed dosimetry verification might be needed to set this treatment on a firmer dosimetric basis. In particular in body areas like the abdomen, adipose tissue and tissue inhomogeneities could complicate accurate modeling of the delivered dose.

While it is a highly valuable goal to develop a method that impacts on cancer cells with high specificity and with minimal adverse effects at its effective dose, it would be very worthwhile to compare the efficacy of this new method with that of other established therapies in suitable animal models. The two primary publications regarding this technology contain animal experiments, but in non-conventional settings: In the 2004 study the authors compared the size of treated vs. sham (wired only) tumors on the same animal at the end of 3-6 days of continuous treatment delivered through implanted wires. This type of study design is problematic because the growth of one tumor can affect the growth of the second tumor, as was elegantly described in papers by Folkman's group [4-7]. In the study from 2007, the authors used a stereotactically implanted rat glioblastoma model, with MRI measurements as a tumor volumetric measurement endpoint, and externally applied electrical fields. Although there appeared to be differences in tumor size, it was unclear how long the tumor growth was followed or whether this treatment prolonged survival of the tumor bearing rats.

A widely accepted endpoint to test the efficacy of anticancer treatments in animal models is the tumor growth delay after application of therapy: the time it takes for control vs. treated tumors to reach a preset volume. This setting allows testing the therapeutic impact of entirely different therapy regimens side-by-side. As the authors point out, the major mechanism of action of TTF is the perturbation of the mitotic spindle for-

\begin{tabular}{|c|c|}
\hline KARGER & (C) 2008 S. Karger GmbH, Freiburg \\
\hline $\begin{array}{l}\text { Fax +497614520714 } \\
\text { E-mail Information@Karger.de } \\
\text { www.karger.com }\end{array}$ & $\begin{array}{l}\text { Accessible online at: } \\
\text { www.karger.com/onk }\end{array}$ \\
\hline
\end{tabular}


mation, similar to what drugs such as paclitaxel would achieve. A side-by-side comparative study of TTF and paclitaxel could be a powerful demonstration of the efficacy, yet relative safety of this new treatment modality. The standard of care for the respective entity is an essential control. In the case of glioblastoma multiforme, one of the main target entities of this application, the current standard of care for previously untreated patients is temozolomide and radiation therapy [8]. NovoCure has recently initiated a phase III trial in which they will compare TTF in recurrent glioblastoma multiforme (GBM) to the current standard of care; in recurrent GBM, this would be chemotherapy (http://www.novocuretrial.com). This is exactly the trial design needed to stringently test the value of this novel therapy in this setting. If successful, the method could eventually be tested in previously untreated populations of patients with GBM, keeping the encouraging safety data in mind.

\section{References}

1 Salzberg M, Kirson E, Palti Y, Rochlitz C: A pilot study with very low-intensity, intermediate-frequency electric fields in patients with locally advanced and/or metastatic solid tumors. Onkologie 2008;31:362-365.

2 Kirson ED, Gurvich Z, Schneiderman R, et al.: Disruption of cancer cell replication by alternating electric fields. Cancer Res 2004;64:3288-3295.

3 Kirson ED, Dbalý V, Tovarys F, et al.: Alternating electric fields arrest cell proliferation in animal tumor models and human brain tumors. Proc Natl Acad Sci U S A 2007;104:10152-10157.
Chen C, Parangi S, Tolentino MJ, Folkman J: A strategy to discover circulating angiogenesis inhibitors generated by human tumors. Cancer Res 1995;55:4230-4233.

5 Folkman J: Angiogenesis inhibitors generated by tumors. Molecular medicine (Cambridge, Mass) 1995;1:120-122.

6 Folkman J: Endogenous inhibitors of angiogenesis. Harvey lectures 1996;92:65-82.
O'Reilly MS, Holmgren L, Shing Y, et al.: Angiostatin: a novel angiogenesis inhibitor that mediates the suppression of metastases by a Lewis lung carcinoma. Cell 1994;79:315-328.

8 Stupp R, Hegi ME, Gilbert MR, Chakravarti A: Chemoradiotherapy in malignant glioma: standard of care and future directions. J Clin Oncol 2007;25: $4127-4136$. 